Research articles

\title{
A novel effect of Electron Spin Resonance on electrical resistivity
}

\author{
Navinder Singh ${ }^{\mathrm{a}}$, Luxmi Rani ${ }^{\mathrm{a}, \mathrm{b}, *}$ \\ ${ }^{a}$ Theoretical Physics Division, Physical Research Laboratory, Ahmedabad 380009, India \\ ${ }^{\mathrm{b}}$ Department of Physics, Bilkent University, 06800 Çankaya, Ankara, Turkey
}

\section{A R T I C L E I N F O}

\section{Keywords:}

Spin relaxation and scattering

Electron paramagnetic resonance

Relaxation

Magnetoresistance

General theory of resonances and relaxations

Theory of electronic transport

Scattering mechanisms

\begin{abstract}
A B S T R A C T
We extend the well known phenomenon of magnetoresistance (extra resistivity of materials in transverse magnetic field) to a regime where in addition to a transverse magnetic field, a transverse microwave field of resonant frequency is also applied. In a magnetic field, electron spin levels are Zeeman split. In a resonant microwave field, we uncover a new channel of momentum relaxation in which electrons in upper Zeeman level can deexcite to lower Zeeman level by generating spin fluctuation excitation in the lattice (similar to what happens in Electron Spin Resonance (ESR) spectroscopy). An additional resistivity due to this mechanism is predicted in which momentum randomization of Zeeman split electrons happen via bosonic excitations (spin fluctuations). An order of magnitude of this additional resistivity is calculated. The whole work is based upon an extension of Einstein's derivation of equilibrium Planckian formula to near equilibrium systems.
\end{abstract}

\section{Introduction}

The phenomenon of magnetoresistance is well studied [1]. Magnetoresistance is an extra resistance of materials in transverse magnetic field. We uncover a hitherto neglected mechanism of resistivity and investigate another "extra" resistance in some specific materials that will appear when alongwith the transverse magnetic field, an microwave field of resonant frequency is applied (Fig. 1). In the figure a sample is placed in a uniform magnetic field which is directed in Zdirection (say). A current is impressed through the sample in the Ydirection. This is the standard set up for magnetoresistance measurements. In addition to the above, we introduce a resonant microwave field along the X-direction (Fig. 1). Thus, a current is passed through the sample which is placed in a criss-crossed static magnetic field and a microwave resonant field of appropriate frequency. The frequency $\omega$ of the microwave field is chosen such that $\hbar \omega=\mu_{B} H_{\text {eff }}$ where $H_{\text {eff }}$ is the effective field "seen" by the conduction electrons in the sample. It is equal to the sum of the external field $(\mathrm{H})$ and any induced internal magnetic field $\left(H_{\text {int }}\right)$ in the sample. The condition $\hbar \omega=\mu_{B} H_{\text {eff }}$ says that the energy of the photon $\hbar \omega$ is resonant with Zeeman splitting of conduction electron energy. This condition is the same as applied in ESR spectroscopy.

In the following development, we will show that this kind of experimental arrangement leads to an "extra" resistance of the sample, and it is related to the microscopic parameters of the material in a fundamental way. Measurement of it can lead to the determination of those parameters. We also determine the order of magnitude of the proposed effect and find that it is well within the current measurements capabilities. We dub this "extra" resistance as Magneto-electro-resistance (generalizing the concept of magnetoresistance). It is to be noted that our proposed effect is fundamentally different from Electrically Detected Magnetic Resonance (EDMR) phenomenon. In EDMR also external magnetic field is applied, and spin states of electrons in semiconductors on donor impurities are flipped by an external microwave source which is made resonant with Zeeman splitting, and thereby enabling them to recombine with conduction holes. This recombination removes charge carriers from the conduction band hence increasing its resistivity [2-18]. Thus in EDMR resistivity changes due to change in carrier concentration in the conduction band (as the conductivity is proportional to carrier concentration). In the present case of extra resistivity carrier concentration remains the same, but a new channel of scattering open up that leads to increased resistivity.

We now proceed to the formulation of the effect and explanation of the mechanism. We have the following physical picture:

- The sample is placed in a magnetic field of strength $\mathrm{H}$ which is directed along Z-direction.

- In transverse direction, a microwave field of frequency " $\omega$ " is applied, such that there is an energy density $u(\omega)$ of the field in the sample.

- Along Y-direction, current I is made to flow by an external field $E$.

\footnotetext{
* Corresponding author.

E-mail addresses: navinder@prl.res.in (N. Singh), luxmiphyiitr@gmail.com (L. Rani).
} 


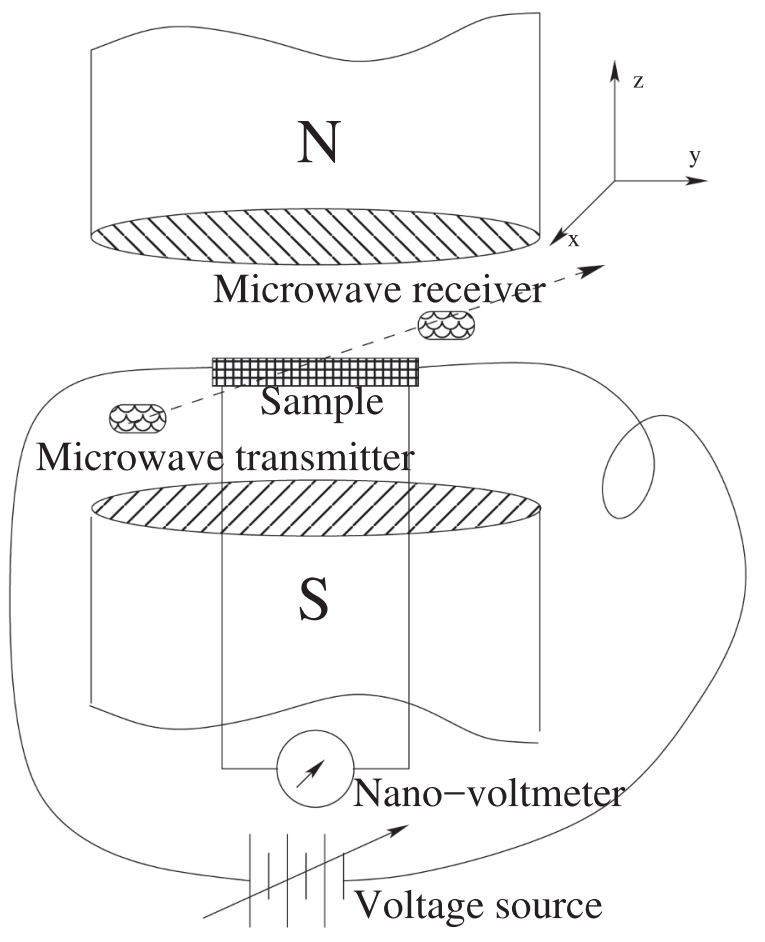

Fig. 1. Schematic diagram of the experimental set-up.

The phenomenological formulation which we put forward goes like this. Under the action of magnetic field, conduction electrons turn into "Itinerant two-level systems" due to Zeeman splitting. And these Itinerant Two-Level Systems (ITLS) drift when external electric field is applied. If a given ITLS is in an upper excited state, it can get rid off an energy $\delta E=E_{2}-E_{1}$ by three processes: (1) by spontaneously giving off a radiation quantum, or (2) by an excitation of a spin fluctuation in the lattice, and (3) by stimulated emission. Similarly, excitation from lower Zeeman level to upper Zeeman level can happen as discussed below. We wish to sketch a calculation along the lines of Einstein's derivation of Planck's formula.

Case-I: Absorption $\left(\left|E_{1}\right\rangle-\left|E_{2}\right\rangle\right)$. The rate at which electrons excite from lower Zeeman level $\left|E_{1}\right\rangle$ to upper Zeeman level $\left|E_{2}\right\rangle$ is given by:

$\frac{d n_{2}}{d t}=B_{12} u(\omega) n_{1}+\alpha \frac{1}{\tau_{\text {spin }}} n_{1}$.

Here, $n_{1}$ is the fraction of Zeeman split electrons per unit volume which are in $\left|E_{1}\right\rangle$ or ground state (Fig. 2). $N_{1}+N_{2}=N$, or $\frac{N_{1}}{N}+\frac{N_{2}}{N}=1$, where $N_{1}$ is the total number of electrons per unit volume in state $\left|E_{1}\right\rangle$, and similarly $N_{2}$ for $\left|E_{2}\right\rangle \cdot n_{i}=\frac{N_{i}}{N}$ is fraction of electrons in ith state. The first term on the R.H.S. of the above equation is the standard Einstein term which says that excitation rate is proportional both to the strength of radiation density $u(\omega)$ and to the number of electrons in the lower level. The proportionality constant is $B_{12}$ (Einstein's $B$ coefficient). In the second term (which we phenomenologically introduce), $1 / \tau_{\text {spin }}$
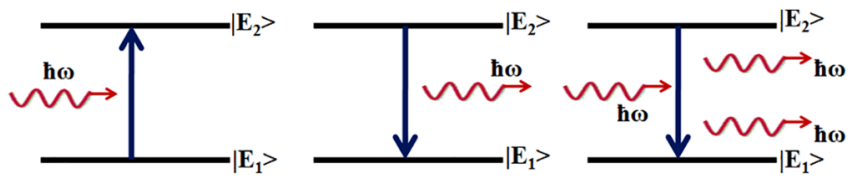

(a) Absorption

\section{(c) Stimulated} Emission
Fig. 2. Schematic diagram of the interaction of an itinerant two level system with field and lattice. represents the collision rate with spin fluctuation in the material. ${ }^{1} \alpha$ is the fraction of collisions that leads to $\left|E_{1}\right\rangle \rightarrow\left|E_{2}\right\rangle$ while 1- $\alpha$ does not lead to $\left|E_{1}\right\rangle \rightarrow\left|E_{2}\right\rangle$, in other words it measures the success rate of upward transition. For a good review of electron spin relxation in metals one can refer to [19].

Case-II: An electron in the upper Zeeman level can de-excite by the following process: (1) spontaneous emission:

$\frac{d n_{1}}{d t}=A_{21} n_{2}$.

here $n_{2}$ is the fraction of Zeeman split electrons per unit volume in upper $\left|E_{2}\right\rangle$ state. $A_{21}$ is the Einstein coefficient of spontaneous emission. (2) Stimulated emission and "spin fluctuation" generation

$\frac{d n_{1}}{d t}=B_{21} u(\omega) n_{2}+\gamma \frac{1}{\tau_{\text {spin }}} n_{2}$.

where $0<\gamma<1$ measures the success rate of the downward transition due to spin fluctuation generation (a spin fluctuation is a bosonic excitation with spin quantum number one, like photon. An electron deexciting from upper level to lower level suffer a change of spin quantum number by one unit, and the generated bosonic excitation also has spin quantum number one. Thus the total spin is conserved in the process, as it should). $B_{21}$ is the Einstein coefficient of induced (or stimulated) emission. In Eqs. (1) and (3), we introduce additional terms $\alpha \frac{1}{\tau_{\text {spin }}} n_{1}$ and $\gamma \frac{1}{\tau_{\text {spin }}} n_{2}$ which take care of transitions induced by spin fluctuation absorption and generation, respectively. Rest of the terms are standard.

In a steady state situation: Absorption $\equiv$ Emission:

$B_{12} u(\omega) n_{1}+\frac{\alpha}{\tau_{\text {spin }}} n_{1}=A_{21} n_{2}+B_{21} u(\omega) n_{2}+\frac{\gamma}{\tau_{\text {spin }}} n_{2}$,

or,

$$
\begin{aligned}
\frac{B_{12} u(\omega)+\frac{\alpha}{\tau_{\text {spin }}}}{A_{21}+B_{21} u(\omega)+\frac{\gamma}{\tau_{\text {spin }}}} & =\frac{n_{2}}{n_{1}} \\
& =\frac{e^{-\beta E_{2}}}{e^{-\beta E_{1}}} \simeq e^{-\beta \hbar \omega} .
\end{aligned}
$$

where $\hbar \omega=E_{2}-E_{1}$ and $\beta=\frac{1}{k_{B} T}$. $k_{B}$ is the Boltzmann constant. We assume that the system remains in a near equilibrium state (a quantitative analysis of this is presented below). On simplifying the above equation we have

$\frac{1}{\tau_{\text {spin }}}\left(\alpha e^{\beta \hbar \omega}-\gamma\right)=A_{21}+B_{21} u(\omega)-B_{12} u(\omega) e^{\beta \hbar \omega}$.

At equilibrium, one must have $\alpha e^{\beta \hbar \omega}=\gamma$. Thus, R.H.S of above equation is zero. Eq. (6) leads to

$u(\omega)=\frac{A_{21}}{B_{12} e^{\beta \hbar \omega}-B_{21}}$.

On applying the standard boundary conditions into Eq. (7); where energy density $u(\omega)$ must tends to infinity $(u(\omega) \rightarrow \infty)$, when $T \rightarrow \infty$; leads to $B_{12}=B_{21}=B$. Thus

$u(\omega)=\frac{A}{B\left(e^{\beta \hbar \omega}-1\right)}$,

which is the Planck's law and the ratio $A / B$ is computed [20] by the Raleigh-Zean limit $\omega \rightarrow 0$.

The situation in which we are interested is a near equilibrium situation. Near equilibrium: $\alpha e^{\beta \hbar \omega} \lesssim \gamma$ (this condition is discussed in the last section), then from Eq. (6) we have

\footnotetext{
${ }^{1}$ In a transport setting electron-phonon interaction is important in simple metals; in magnetic metals electron-spin scattering becomes an important interaction like in $\mathrm{MnSi}, \mathrm{ZrZn}_{2}$.
} 
$\frac{1}{\tau_{\text {spin }}}=B u(\omega)\left(\frac{e^{\beta \hbar \omega}-1}{\gamma-\alpha e^{\beta \hbar \omega}}\right)-A\left(\frac{1}{\gamma-\alpha e^{\beta \hbar \omega}}\right)$.

This is an important result in which spin fluctuation scattering rate is expressed in terms of phenomenon parameters $\alpha, \gamma, u(\omega), A$, and $B$.

\section{Electro-magneto resistance using the drude theory}

To calculate the extra resistance due to spin fluctuation generation and absorption we proceed with the simple Drude theory of momentum relaxation. This is similar to the solution of the Boltzmann equation under relaxation time approximation [21]. The Drude theory is characterized by a constant momentum relaxation rate $1 / \tau$. If $\vec{P}$ is an average momentum of an electron, then the equation of motion under various relaxation processes is given by:

$\frac{d \overrightarrow{\mathrm{P}}}{d t}=-e \overrightarrow{\mathrm{E}}-\frac{1}{\tau_{\text {others }}} \overrightarrow{\mathrm{P}}-\frac{\alpha}{\tau_{\text {spin }}} n_{1} \overrightarrow{\mathrm{P}}-\frac{\gamma}{\tau_{\text {spin }}} n_{2} \overrightarrow{\mathrm{P}}$

Here $\frac{1}{\tau_{\text {others }}}$ is the standard Drude relaxation rate due to impurity scattering. ${ }^{2}$ We introduce two more relaxation terms: $-\frac{\alpha}{\tau_{\text {spin }}} n_{1} \overrightarrow{\mathrm{P}}$ and $-\frac{\gamma}{\tau_{s p i n}} n_{2} \overrightarrow{\mathrm{P}}$. The first term denotes momentum randomization of an electron when it absorbs a spin fluctuation quantum (as explained below Eq. (1)). The second term (latter term) denotes the momentum randomization of a drifting electron when it generates a spin fluctuation quantum and thereby de-excites from the higher Zeeman level to the lower Zeeman level. The momentum randomization happens as the direction of these excitations is random (much like what happens in the case of spontaneous emission, in which an atom suffer random kicks due to spontaneously emitted photons). As we are considering a near equilibrium situation we have $\frac{n_{2}}{n_{1}}=e^{-\beta \hbar \omega}$, where $n_{1}=$ $\frac{e^{\beta \hbar \omega}}{e^{\beta \hbar \omega}+1}, n_{2}=\frac{1}{e^{\beta \hbar \omega}+1}, n_{1}+n_{2}=1$. The equation of motion (Eq. (10)) takes the form:

$\frac{d \overrightarrow{\mathrm{P}}}{d t}=-e \overrightarrow{\mathrm{E}}-\overrightarrow{\mathrm{P}}\left(\frac{1}{\tau_{\text {others }}}+\frac{1}{\tau_{\text {spin }}}\left(\frac{\alpha e^{\beta \hbar \omega}+\gamma}{e^{\beta \hbar \omega}+1}\right)\right)$

In the Ohmic regime $\vec{J}=\sigma \vec{E}=-e n \vec{V}$, and $\vec{P}=m \vec{V}$. Thus, the resistivity $(\rho=1 / \sigma)$ is

$\rho=\frac{m}{n_{e} e^{2}}\left(\frac{1}{\tau_{\text {other }}}+\frac{1}{\tau_{\text {spin }}}\left(\frac{\alpha e^{\beta \hbar \omega}+\gamma}{e^{\beta \hbar \omega}+1}\right)\right)$

Here, the second term is additional resistivity with

$\frac{1}{\tau_{\text {additional }}}=\left(\frac{\gamma+\alpha e^{\beta \hbar \omega}}{\gamma-\alpha e^{\beta \hbar \omega}}\right) \times\left(B u(\omega) \tanh \left(\frac{1}{2} \beta \hbar \omega\right)-\frac{A}{e^{\beta \hbar \omega}+1}\right)$

where we used the Eq. (9). Thus the additional resistivity is given by

$$
\begin{aligned}
\rho_{\text {additional }}= & \frac{m}{n_{e} e^{2}} \frac{1}{\tau_{\text {additional }}}=\frac{m}{n_{e} e^{2}}\left(\frac{\gamma+\alpha e^{\beta \hbar \omega}}{\gamma-\alpha e^{\beta \hbar \omega}}\right) \\
& \times\left(B u(\omega) \tanh \left(\frac{1}{2} \beta \hbar \omega\right)-\frac{A}{e^{\beta \hbar \omega}+1}\right) .
\end{aligned}
$$

This is the central result of the present investigation. Thus there must be an "extra" resistivity solely coming from the excitation and absorption of spin fluctuations in the lattice whose quantum is equal to the separation between the Zeeman levels of the drifting electron. The importance of this effect is that it can be externally tuned by controlling the Zeeman splitting through external magnetic field. Spontaneous rate in condensed matter systems is generally weak. So the result simplifies to:

\footnotetext{
${ }^{2}$ The static part $-e V \times B$ is not contributing as the transverse Lorentz force $-e V \times B$ is counter-balanced by the induced Hall electric field in the steady state condition.
}

$\rho_{\text {additional }}=\frac{m}{n_{e} e^{2}} B u(\omega)\left(\frac{\gamma+\alpha e^{\beta \hbar \omega}}{\gamma-\alpha e^{\beta \hbar \omega}}\right) \tanh \left(\frac{1}{2} \beta \hbar \omega\right)$

\section{Typical values and the order of magnitude of the effect}

To roughly estimate of the order of magnitude of the effect we take values for a typical metallic sample which is placed in a magnetic field of 1 Tesla (for example) and at temperature of $100 \mathrm{~K}\left(\mu_{B} H=\hbar \omega\right)$. Let us take $B u(\omega) \simeq 10^{10} \mathrm{~Hz}$ (refer to footnote. ${ }^{3}$ )

The spontaneous transition rate of a Zeeman spit electron from upper level to lower level in metals is of the order of MHz. ${ }^{4}$ Thus, we can safely neglect its contribution to additional scattering rate. We assume that $\frac{\gamma}{\alpha} \simeq 1.01$. The regime corresponding to this ratio is investigated below. With this ratio one is not far from equilibrium as shown below. Having this input of the physical parameters, the additional scattering rate (from Eq. (13)) turns out to be: where $\tanh (x) \sim x$ for small $x$. The extra resistivity due to this is given by $\rho_{\text {extra }}=\frac{m}{n_{e} e^{2}}\left(1 / \tau_{\text {add }}\right) \simeq 10^{-9} \Omega \cdot$ meters (we take $n_{e}=10^{26}$ per meter cube (typical electron number density in metals)). ${ }^{5}$

If we have a sample of a size $4 \mathrm{~mm} \times 4 \mathrm{~mm} \times 4 \mathrm{~mm}$, then the extra resistance of it will be $\sim 1$ micro $\Omega$. This should not be difficult to measure. If a current of $100 \mathrm{~mA}$ is made to flow in the sample, then change in the voltage drop (due to change in the resistance $\Delta V=I \Delta R$ ) will be 100 nano-volts, which again can be detected with a sensitive nano-voltmeter.

\section{The operational regime}

We now investigate what is meant by $\gamma \gtrsim \alpha e^{\beta \hbar \omega}$ (the near equilibrium condition) [22]. We neglect the weaker spontaneous emission term. We consider the two cases separately:

Case (1) External fields only:

$\frac{d n_{1}}{d t}=n_{2} B_{21} u(\omega)-n_{1} B_{12} u(\omega)$.

Here, let us set $n=n_{1}-n_{2}$ and $n_{1}+n_{2}=1$ (as before $n_{i}=\frac{N_{i}}{N}$ ). Then, we have

$\frac{1}{2} \frac{d n}{d t}=-n B u(\omega)$

After simplification, we get the relation;

$n(t)=n(0) e^{-2 B u(\omega) t}$.

Power absorbed by the sample from the field:

$P_{\text {absorbed }}=n_{1} N B u(\omega) \hbar \omega-n_{2} N B u(\omega) \hbar \omega=n N B u(\omega) \hbar \omega$.

Case (2): Spin-fluctuation scattering only:

$\frac{d n_{1}}{d t}=n_{2}\left(\frac{\gamma}{\tau_{s}}\right)-n_{1}\left(\frac{\alpha}{\tau_{s}}\right)$

\footnotetext{
${ }^{3}$ From Fermi golden rule, it can be roughly approximated as: $\frac{1}{\tau} \simeq \frac{2 \pi}{\hbar}|V|^{2} \rho(E)$, where $\rho(E) \sim a^{-3}, a$ is the lattice constant $(a \sim 1 \AA)$ and $\mathrm{V}$ is in sub- $\mu$ eV range. Thus $\frac{1}{\tau} \sim 10^{10}$ in per sec.

${ }^{4}$ This can be inferred from the ESR linewidths which are in $\mathrm{MHz}$ range. The linewidths have contributions from interactions in the material along with spontaneous rate.

${ }^{5}$ For comparison purpose, typical value of magnetoresistance in metals is of the order of several nano-Ohms-meter. For example, metal silver has resistivity of about $5 \times 10^{-10} \Omega-$ meters at low temperature of 4 Kelvin, and its magnetoresistance, at that temperature and in magnetic field of about $2 T$, is about $5 \times 10^{-9} \Omega-$ meters. Thus the extra resistivity would be of the order of magneto-resistance (it will be in addition to that thus measurable. One can switch on or off the microwave field to see this effect).
} 


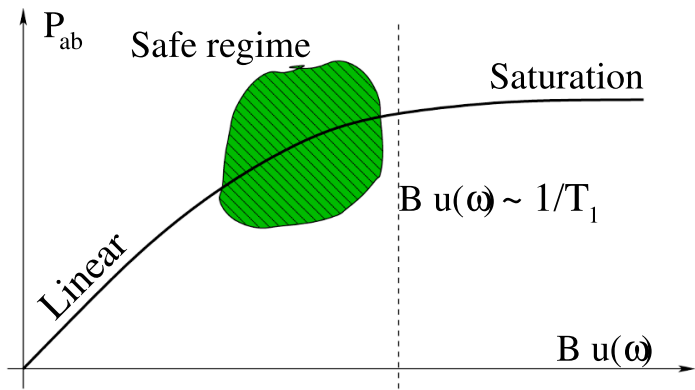

Fig. 3. Schematic depiction of the intermediate regime (green shaded area) in which the system is to be forced by tunning $u(\omega)$.

In the equilibrium state: $\frac{d n_{1}}{d t}=0$, thus

$\frac{n_{2}}{n_{1}}=\frac{\alpha}{\gamma}=e^{-\beta \hbar \omega}$,

or, $\alpha e^{\beta \hbar \omega}=\gamma$ is the equilibrium condition.

From Eq. (20), we have

$T_{1} \frac{d n}{d t}=n_{0}-n$,

where $T_{1}=\frac{\tau_{S}}{\alpha+\gamma}$ (as usually defined in the NMR spectroscopy) and $n_{0}=\frac{\gamma-\alpha}{\gamma+\alpha}$.

$n(t)=n_{0}\left(1-e^{-t / T 1}\right)$.

When both are acting together in a steady state situation

$\left(\frac{d n}{d t}\right)_{\text {spin-fluc }}+\left(\frac{d n}{d t}\right)_{\text {field }}=0$,

thus

$n(t)=\frac{n_{0}}{1+2 B u(\omega) T_{1}}$.

There are two possible cases:

Case A: If $2 B u(\omega) T_{1} \ll 1$ or $T_{1} \ll \frac{1}{2 B u(\omega)}\left(\frac{\alpha+\gamma}{\tau_{s}} \gg 2 B u(\omega)\right) . n(t) \simeq n_{0}$. This means Spin scattering is fast, system stays in equilibrium.

Case B: If $2 B u(\omega) T_{1} \gg 1$ or $\left(\frac{\alpha+\gamma}{\tau_{s}} \ll 2 B u(\omega)\right)$. This means strong field and system stays away from equilibrium.

\section{Power absorbed}

$P_{a b}=n N B u(\omega) \hbar \omega=\frac{n_{0} \hbar \omega B u(\omega)}{1+2 B u(\omega) T_{1}}$.

The regime which we consider is the intermediate one (Fig. (3)). In the example numerical case mentioned above, the power absorbed is in micro-watts.

\section{Conclusion}

The mechanism of spin wave generation when a Zeeman split electron makes a downward transition from upper Zeeman level to lower Zeeman level leads to random kicking of the conduction electron. This random kicking is the source of momentum randomization of the electron and leads to extra resistivity which we uncover. Similarly, the absorption of spin waves leads to random kicking and extra resistance. We calculate an order of magnitude of this effect and we find that it is well within the detectable limits.

\section{Acknowledgement}

We thank Rajesh Kumar Kushawaha, Prashant Kumar, K. P. Subramanian and Gopalakrishnan Balasubramanian for useful suggestions and discussions.

\section{References}

[1] A.B. Pippard, Magnetoresistance in metals, CUP, Cambridge, UK (1989); R. G. Chambers, Electrons in Metals and Semiconductors, Springer, Netherlands (1990).

[2] R.N. Ghosh, R.H. Silsbee, Phys. Rev. B 46 (1992) 12508.

[3] L.S. Vlasenko, Yu.V. Martynov, T. Gregorkiewicz, C.A.J. Ammerlaan, Phys. Rev. B 52 (1995) 1149.

[4] C.F.O. Graeff, M.S. Brandt, M. Stutzmann, M. Holzmann, G. Abstreiter, F. Schäffler, Phys. Rev. B 59 (1999) 242.

[5] O.M. Fedorych, Z. Wilamowski, W. Jantsch, J. Sadowski, Acta Phys. Pol., A 105 (2004) 591.

[6] C. Boehme, H. Malissa, eMagRes 6 (2017) 83.

[7] A.R. Stegner, C. Boehme, H. Huebl, M. Stutzmann, K. Lips, M.S. Brandt, Nature Phys. 2 (2006) 835.

[8] D.R. McCamey, H. Huebl, M.S. Brandt, W.D. Hutchison, J.C. McCallum, R.G. Clark, A.R. Hamilton, Appl. Phys. Lett. 89 (2006) 182115

[9] C.C. Lo, V. Lang, R.E. George, J.J.L. Morton, A.M. Tyryshkin, S.A. Lyon, J. Bokor, T. Schenkel, Phys. Rev. Lett. 106 (2011) 207601.

[10] C. Boehme, K. Lips, Phys. Rev. B 68 (2003) 245105 .

[11] L. Zhu, K.J. van Schooten, M.L. Guy, C. Ramanathan, Phys. Rev. Appl. 7 (2017) 064028 .

[12] S.-Y. Lee, S. Paik, D.R. McCamey, C. Boehme, Phys. Rev. B 86 (2012) 115204

[13] S.-Y. Lee, S. Paik, D.R. McCamey, C. Boehme, Phys. Rev. B 86 (2012) 115204 .

[14] F. Hoehne, L. Dreher, J. Behrends, M. Fehr, H. Huebl, K. Lips, A. Schnegg, M. Suckert, M. Stutzmann, M.S. Brandt, Rev. Sci. Instrum. 83 (2012) 043907

[15] D.P. Franke, F. Hoehne, L.S. Vlasenko, K.M. Itoh, M.S. Brandt, Phys. Rev. B 89 (2014) 195207 .

[16] L. Dreher, F. Hoehne, H. Morishita, H. Huebl, M. Stutzmann, K.M. Itoh, M.S. Brandt, Phys. Rev. B 91 (2015) 075314.

[17] T.L. Keevers, W.J. Baker, D.R. McCamey, Phys. Rev. B 91 (2015) 205206 .

[18] J. Behrends, A. Schnegg, K. Lips, E.A. Thomsen, A.K. Pandey, I.D.W. Samuel, D.J. Keeble, Phys. Rev. Lett. 105 (2010) 176601

[19] J. Fabian, S. Das Sharma, J. Vac. Sci. Technol. B 17 (1999) 1708.

[20] H. Haken, H.C. Wolf, The Physics of Atoms and Quanta, Springer Verlag, Berlin, Heidelberg, 2004;

R. Eisberg, R. Resnick, Quantum Physics, John Wiley and Sons, New York, 1985.

[21] N. Singh, Electronic Transport Theories: From Weakly to Strongly Correlated Materials, CRC Press, Taylor and Francis group, LLC, UK, 2017.

[22] C.P. Slichter, Principles of Magnetic Resonance, Springer-Verlag, Berlin, Heidelberg, 1990. 